\title{
Combined analysis of charm-quark fragmentation-fraction measurements
}

\author{
Mykhailo Lisovyi $^{a}$, Andrii Verbytskyi ${ }^{b}$ and Oleksandr Zenaiev ${ }^{* c}$ \\ a Physikalisches Institut der Universität Heidelberg \\ ${ }^{b}$ Max-Planck-Institut für Physik \\ ${ }^{c} D E S Y$ \\ E-mail: mikhaylo.lisovyi@desy.de, andrii.verbytskyi@mpp.mpg.de, \\ oleksandr.zenaievedesy.de
}

A summary of measurements of the fragmentation of charm quarks into a specific hadron is given. Measurements performed in photoproduction and deep inelastic scattering in $e^{ \pm} p, p p$ and $e^{+} e^{-}$collisions are compared, using up-to-date branching ratios. Within uncertainties, all measurements agree, supporting the hypothesis that fragmentation is independent of the specific production process. Compared to individual measurements, the averaged fragmentation fractions have significantly reduced uncertainties. Implications of results for other physical quantities are considered.

XXIV International Workshop on Deep-Inelastic Scattering and Related Subjects

11-15 April, 2016

DESY Hamburg, Germany

\footnotetext{
* Speaker.
} 


\section{Introduction}

The fragmentation process is one of the most important phenomena in the high-energy physics. This process is soft and hence can not be calculated with the techniques of perturbative QCD (pQCD), so it is important to improve the knowledge about the process and study the most basic properties of the fragmentation. One of these is the probability of the fragmented quark $q$ to create a given hadron $H_{q}$. This is called the fragmentation fraction and is denoted below as $f\left(q \rightarrow H_{q}\right)$.

An important question is whether the fragmentation fractions are universal, i.e. independent of the hard production mechanism. If true, once precisely measured in one environment, these can be applied in any other. This is especially important for the studies of heavier $c$ and $b$ quarks, as the precise knowledge of $f\left(c \rightarrow H_{c}\right)$ and $f\left(b \rightarrow H_{b}\right)$ can help for many measurement in the modern high-energy physics experiments.

The goal of this analysis $[1,2]$ is to provide the most precise and model-independent values for fragmentation fractions of the charm quark, simultaneously testing the hypothesis that the fragmentation fractions are universal and the sum of them is equal to unity, thus checking if all weakly decaying states are known. To check the former, we extract the fragmentation fractions from all measurements simultaneously and separately for different environments and compare the results. To check the latter, the Standard Model predictions for the total charm cross-section in $e^{+} e^{-}$collisions are utilisied.

\section{Selection of measurements for the extraction of fragmentation fractions}

The selection of the measurements for the extraction of fragmentation fractions was done according to a set of criteria explained below. First, the selection is limited to the measurements obtained in the collisions of particle beams as these environments have well know production mechanisms of charm quarks and assure an absence of possible matter effects. Thus, only the measurements from high-energy $e p, p p$ and $e^{+} e^{-}$collisions satisfy the requirements. The second criterion is the precision of the measured quantities. Basically, only the experiments on LEP, TEVATRON, HERA, LHC and $B$-factories provide sufficient and competitive precision. The third criterion of the selection is the sufficient number of measurements in the given physical environment, otherwise they cannot be treated independently and/or constrain the fragmentation fractions.

The measurements selected according to the criteria described above form five groups: from $B$ factories [3-9], $Z$ decays at LEP [10-14], deep inelastic scattering in $e^{ \pm} p$ collisions at HERA [1517], photoproduction at HERA [18,19] and $p p$ collisions at the LHC [20-25]. To make separate inputs consistent, the original measurements are corrected to the same up-to-date world averages of branching ratios from Ref. [26].

\subsection{Calculation of the fragmentation fractions and combination procedure}

In this analysis the charm-quark fragmentation fraction to a specific hadron assumed to be equal to the ratio of the production cross-section of the hadron via charm quark over the production cross-section of the charm quark

$$
f\left(c \rightarrow H_{c}\right)=\sigma\left(H_{c}\right) / \sigma(c) .
$$


With the available precise predictions for the total charm cross-section in $e^{+} e^{-}$collisions it is possible to calculate $f\left(c \rightarrow H_{c}\right)$ according to Eq. (2.1). However, sufficiently precise predictions for the charm-quark production in $p p$ and $e^{ \pm} p$ collisions are not available and other approaches are needed. Hereby, we make an assumption that the sum of charm-quark fragmentation fractions to all known ground states of charm hadrons is unity, the charm-quark fragmentation fraction to a specific hadron can be calculated as the ratio of the hadron-production cross-section over the sum of cross-sections of all known ground states of charm hadrons

$$
f\left(c \rightarrow H_{c}\right)=\sigma\left(H_{c}\right) / \Sigma_{i} \sigma\left(H_{c, i}\right) .
$$

The fragmentation fractions calculated according to Eq. (2.1) for the $e^{+} e^{-}$collisions and $Z$ decays allow an independent check that the sum of the fragmetation fractions of all weakly decaying charm states:

$$
S=f\left(c \rightarrow D^{0}\right)+f\left(c \rightarrow D^{+}\right)+f\left(c \rightarrow D_{s}^{+}\right)+f\left(c \rightarrow \Lambda_{c}^{+}\right)+\sum f\left(c \rightarrow \Xi_{c}^{+, 0}, \Omega_{c}^{0}\right)
$$

is close to unity with sufficient accuracy. To perform the check we combine the available measurements of $D^{0}, D^{+}, D_{s}^{+}, \Lambda_{c}^{+}$and assume $\sum f\left(c \rightarrow \Xi_{c}^{+, 0}, \Omega_{c}^{0}\right)=\lambda f\left(c \rightarrow \Lambda_{c}^{+}\right)$, where $\lambda \ll 1$ is estimated from the production rates of strange hadrons.

The combination of the measurements used in the present analysis is based on numerical $\chi^{2}$ minimisation with respect to observables of interest. The numerical minimisation was performed with the MINUIT package [30] and the procedure for calculation of $\chi^{2}$ itself is outlined in Ref. [1].

The obtained fragmentation fractions are also used to calculate the quantities commonly exploited as Monte Carlo generator parameters:

$$
\begin{gathered}
R_{u / d}=\frac{f\left(c \rightarrow D^{0}\right)-f\left(c \rightarrow D^{*+}\right) \mathscr{B}_{D^{*+} \rightarrow D^{0}}}{f\left(c \rightarrow D^{+}\right)+f\left(c \rightarrow D^{*+}\right) \mathscr{B}_{D^{*+} \rightarrow D^{0}}} \\
\gamma_{s(1)}^{(*)}=\frac{2 f\left(c \rightarrow D_{s(1)}^{(*)+}\right)}{f\left(c \rightarrow D^{(*)+}\right)+f\left(c \rightarrow D^{(*) 0}\right)} \text { and } P_{V}^{d}=\frac{f\left(c \rightarrow D^{*+}\right)+f\left(c \rightarrow D^{* 0}\right)}{f\left(c \rightarrow D^{+}\right)+f\left(c \rightarrow D^{0}\right)} .
\end{gathered}
$$

\section{Results}

The results of the fragmentation fraction extraction procedure for each of the groups introduced above is shown in Fig. 1. The numerical values can be found in Ref. [1].

To check the consistency of the data from different production environments and also to extract the charm-quark fragmentation fractions with high precision, all input measurements are used together to produce a global combination. The constraint on the sum of the cross-sections of the weakly decaying charm states, $S$, is imposed in the combination, i.e. the prediction for the total charm cross-sections in $e^{+} e^{-}$collisions is not used, in order to minimise model dependence of the averaging procedure. The result of averaging $e^{+} e^{-}, e^{ \pm} p$ and $p p$ data, with the constraint $S=1$ is presented in the middle column of Tab. 1 and is shown in Fig. 1. ${ }^{1}$ The input data are in very good

\footnotetext{
${ }^{1}$ As an alternative, the combination is also performed using both the constraint on $S$ as well as theoretical predictions of charm production in $e^{+} e^{-}$collisions and $Z$ decays; the result of the averaging procedure with this approach is given
} 

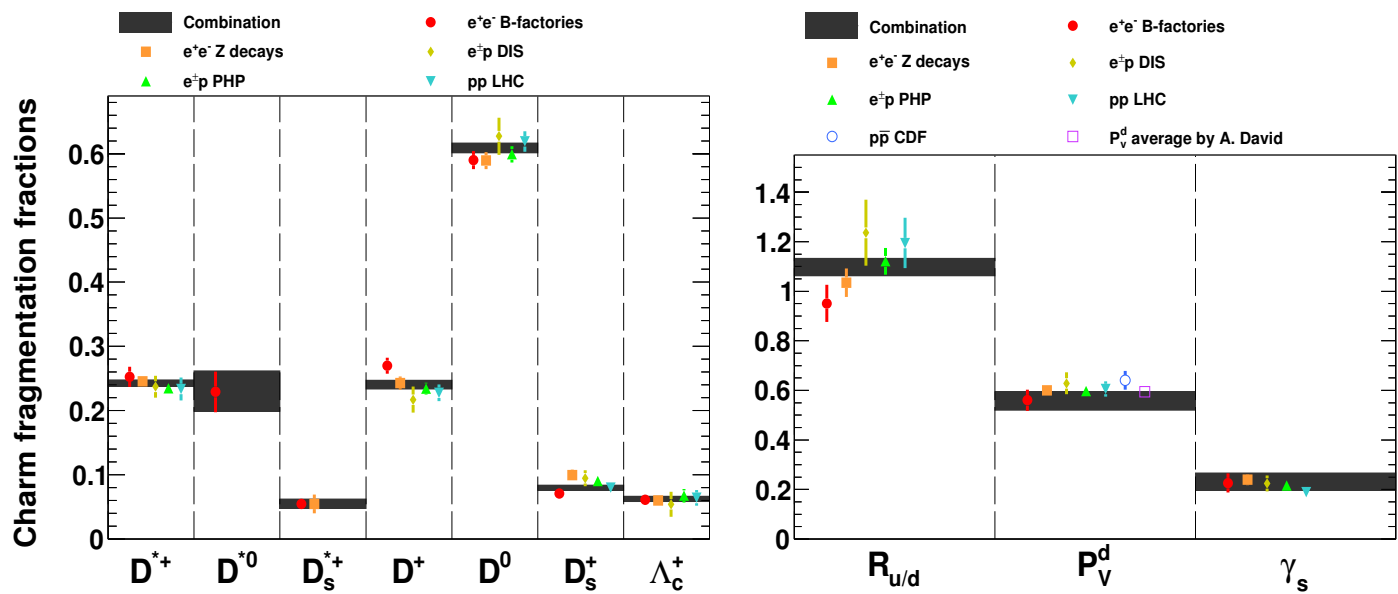

Figure 1: The values of charm-quark fragmentation fractions, $f\left(c \rightarrow H_{c}\right)$ (left), and $R_{u / d}, P_{V}^{d}, \gamma_{s}$ (right) in different experiments with the $S$ constraint. The global combination with the $S$ constraint is shown with the shaded band. Averages of included data in different production environments are shown with various full symbols. Data that were not included in the combination [20-22,31-33] are shown with open symbols. Note, that the latter are quoted from the original papers, i.e. without correction to the up-to-date branching ratios and with no branching ratio uncertainty, if not given in the source.

Table 1: Results of the global combination.

\begin{tabular}{|c|c|c|}
\hline & Constrained $S$ & $\begin{array}{c}\text { Constrained } S, \text { fix } \\
\sigma\left(e^{+} e^{-} \rightarrow c \bar{c}\right), \frac{\Gamma_{c \bar{c}}}{\Gamma_{\text {hadrons }}} .\end{array}$ \\
\hline \hline$f\left(c \rightarrow D^{*+}\right)$ & $0.2430 \pm 0.0049$ & $0.2386 \pm 0.0046$ \\
\hline$f\left(c \rightarrow D^{* 0}\right)$ & $0.2305 \pm 0.0315$ & $0.2251 \pm 0.0299$ \\
\hline$f\left(c \rightarrow D_{s}^{*+}\right)$ & $0.0547 \pm 0.0074$ & $0.0536 \pm 0.0072$ \\
\hline$f\left(c \rightarrow D^{+}\right)$ & $0.2404 \pm 0.0067$ & $0.2439 \pm 0.0067$ \\
\hline$f\left(c \rightarrow D^{0}\right)$ & $0.6089 \pm 0.0076$ & $0.6143 \pm 0.0073$ \\
\hline$f\left(c \rightarrow D_{s}^{+}\right)$ & $0.0799 \pm 0.0040$ & $0.0794 \pm 0.0040$ \\
\hline$f\left(c \rightarrow \Lambda_{c}^{+}\right)$ & $0.0623 \pm 0.0041$ & $0.0548 \pm 0.0026$ \\
\hline \hline$\chi^{2}$ & 65.7 & 87.1 \\
\hline$n_{\text {dof }}$ & 64 & 67 \\
\hline \hline$S$ & $1.0000 \pm 0.0005$ & $1.0000 \pm 0.0004$ \\
\hline$R_{u / d}$ & $1.0976 \pm 0.0354$ & $1.1168 \pm 0.0354$ \\
\hline$P_{V}^{d}$ & $0.5575 \pm 0.0375$ & $0.5402 \pm 0.0355$ \\
\hline$\gamma_{s}$ & $0.1881 \pm 0.0103$ & $0.1851 \pm 0.0101$ \\
\hline$\gamma_{s}^{*}$ & $0.2311 \pm 0.0346$ & $0.2313 \pm 0.0346$ \\
\hline
\end{tabular}

agreement with $\chi^{2} / n_{\mathrm{dof}}=65.7 / 64$. The result of the combination has significantly reduced uncertainties compared to individual measurements. The values of the charm production cross-sections in $p p$ collisions, obtained in the global fit, are consistent with those obtained in the original analysis and have significantly reduced uncertainties as well. The consistent treatment of the LHCb and ALICE measurements in the combination procedure allows unbiased calculation of the ratio of the inclusive fiducial charm-quark production cross-sections at different centre-of-mass energies and in the right column of Tab. 1. 
Table 2: Results of the global combination for $L=1$ states.

\begin{tabular}{|c|c|}
\hline & Average \\
\hline \hline$f\left(c \rightarrow D_{1}^{+}\right)$ & $0.0460_{-0.0182}^{+0.0269}$ \\
\hline$f\left(c \rightarrow D_{2}^{*+}\right)$ & $0.0320_{-0.0082}^{+0.0094}$ \\
\hline$f\left(c \rightarrow D_{1}^{0}\right)$ & $0.0297 \pm 0.0038$ \\
\hline$f\left(c \rightarrow D_{2}^{* 0}\right)$ & $0.0394 \pm 0.0068$ \\
\hline$f\left(c \rightarrow D_{s 1}^{+}\right)$ & $0.0109 \pm 0.0014$ \\
\hline \hline$\gamma_{s 1}$ & $0.287_{-0.109}^{+0.079}$ \\
\hline
\end{tabular}

their comparison to theoretical predictions as discussed in Ref. [1].

In addition to the average fragmentation fractions for the ground, $L=0$, states, some fragmentation fractions for the excited, $L=1$ charm hadrons are calculated. The measurements used for the averaging are obtained by ZEUS [34,35], OPAL [36] and ALEPH [37] experiments. The unpublished measurement of $f\left(c \rightarrow D_{s 1}^{+}\right)$from Ref. [38] was not used. As the measurements have limited precision, the correction to the most recent branching ratios was not needed. The averages, as well as the $\gamma_{s 1}$, are calculated with an assumption of fully uncorrelated statistical and systematical uncertainties. The results are given in Tab. 2.

\section{Summary}

A summary of measurements of the fragmentation of charm quarks into a specific charm hadron is given. The analysis includes data collected in photoproduction and deep inelastic scattering in $e^{ \pm} p$ collisions and well as $e^{+} e^{-}$and $p p$ data. Measurements in different production regimes agree within uncertainties, supporting the hypothesis that fragmentation proceeds independent of the specific production process. Averages of the fragmentation fractions are presented. The global average has significantly reduced uncertainties compared to individual measurements. In addition, the hypothesis that the sum of fragmentation fractions of all known weakly decaying charm hadrons is equal to unity is checked to hold within 3 standard deviations using the $e^{+} e^{-}$data. The obtained results should be applied for the best precision of relevant measurements and theoretical predictions.

\section{Acknowledgements}

We thank Erich Lohrmann for his major contribution to the development of this analysis. We thank Uri Karshon and Stefan Kluth for useful discussions and help in the work with the bibliography. We also thank Alexander Glazov and Ian Brock for the critical reading of the analysis manuscript [1] and useful suggestions on text improvement.

\section{References}

[1] M. Lisovyi, A. Verbytskyi and O. Zenaiev (2015), 1509.01061v1

[2] M. Lisovyi, A. Verbytskyi and O. Zenaiev, Combined analysis of charm-quark fragmentation-fraction measurements, in proceedings of XLV International Symposium on Multiparticle Dynamics (2015) 
[3] D. Bortoletto et al. (CLEO Collaboration), Phys.Rev. D37, 1719 (1988)

[4] P. Avery et al. (CLEO Collaboration), Phys.Rev. D43, 3599 (1991)

[5] H. Albrecht et al. (ARGUS Collaboration), Z.Phys. C52, 353 (1991)

[6] H. Albrecht et al. (ARGUS Collaboration), Z.Phys. C54, 1 (1992)

[7] H. Albrecht et al. (ARGUS Collaboration), Phys.Lett. B207, 109 (1988)

[8] R. Seuster et al. (Belle Collaboration), Phys.Rev. D73, 032002 (2006), hep-ex / 0506068

[9] B. Aubert et al. (BaBar Collaboration), Phys.Rev. D75, 012003 (2007), hep-ex/ 0609004

[10] G. Alexander et al. (OPAL Collaboration), Z.Phys. C72, 1 (1996)

[11] K. Ackerstaff et al. (OPAL Collaboration), Eur.Phys.J. C1, 439 (1998), hep-ex/ 9708021

[12] R. Barate et al. (ALEPH Collaboration), Eur.Phys.J. C16, 597 (2000), hep-ex/ 9909032

[13] P. Abreu et al. (DELPHI Collaboration), Eur.Phys.J. C12, 225 (2000)

[14] P. Abreu et al. (DELPHI Collaboration), Eur.Phys.J. C12, 209 (2000)

[15] S. Chekanov et al. (ZEUS Collaboration), JHEP 0707, 074 (2007), 0704.3562

[16] H. Abramowicz et al. (ZEUS Collaboration), JHEP 1011, 009 (2010), 1007.1945

[17] A. Aktas et al. (H1 Collaboration), Eur.Phys.J. C38, 447 (2005), hep-ex/ 0408149

[18] S. Chekanov et al. (ZEUS Collaboration), Eur.Phys.J. C44, 351 (2005), hep-ex/ 0508019

[19] H. Abramowicz et al. (ZEUS Collaboration), JHEP 1309, 058 (2013), 1306.4862

[20] B. Abelev et al. (ALICE Collaboration), JHEP 1201, 128 (2012), 1111.1553

[21] B. Abelev et al. (ALICE Collaboration), Phys.Lett. B718, 279 (2012), 1208 . 1948

[22] B. Abelev et al. (ALICE Collaboration), JHEP 1207, 191 (2012), 1205.4007

[23] G. Aad et al. (ATLAS Collaboration), Nucl. Phys. B907, 717 (2016), 1512.02913

[24] R. Aaij et al. (LHCb Collaboration), Nucl.Phys. B871, 1 (2013), 1302.2864

[25] R. Aaij et al. (LHCb Collaboration), Submitted to JHEP (2015), 1510.01707

[26] K.A. Olive et al. (Particle Data Group), Chin.Phys. C38, 090001 (2014 and 2015 update)

[27] A. Zupanc et al. (Belle Collaboration), Phys.Rev.Lett. 113, 042002 (2014), 1312 . 7826

[28] M. Ablikim et al. (BESIII Collaboration), Phys.Rev. D91, 031101 (2015), 1412.4566

[29] B. Aubert et al. (BaBar Collaboration), Phys.Rev. D72, 091101 (2005), hep-ex/ 0508039

[30] F. James and M. Roos, Comput.Phys.Commun. 10, 343 (1975)

[31] G. Aad et al. (ATLAS Collaboration), ATLAS preliminary ATLAS-CONF-2011-017 (2011)

[32] D. Acosta et al. (CDF Collaboration), Phys.Rev.Lett. 91, 241804 (2003), hep-ex/ 0307080

[33] A. David, Phys.Lett. B644, 224 (2007)

[34] H. Abramowicz et al. (ZEUS Collaboration), Nucl.Phys. B866, 229 (2013), 1208.4468

[35] S. Chekanov et al. (ZEUS Collaboration), Eur.Phys.J. C60, 25 (2009), 0807.1290

[36] K. Ackerstaff et al. (OPAL Collaboration), Z.Phys. C76, 425 (1997)

[37] A. Heister et al. (ALEPH Collaboration), Phys.Lett. B526, 34 (2002), hep-ex/ 0112010

[38] A. Verbytskyi, Ph.D. thesis, Universität Hamburg (2013) 